NASA CONTRACTOR REPORT
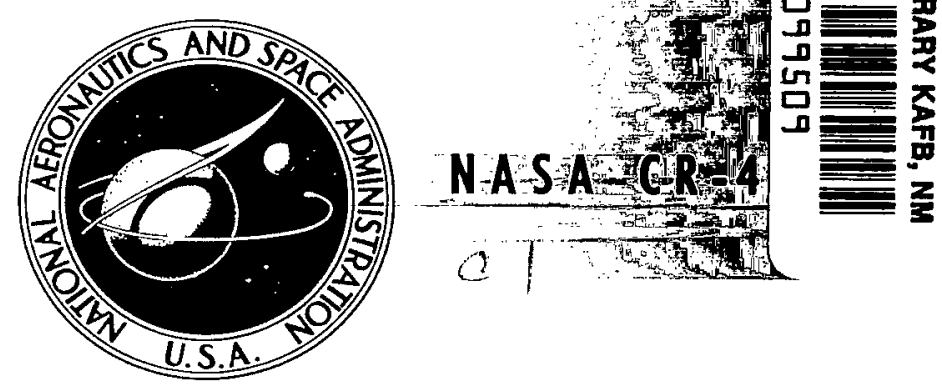

0
0
0
0
1
0
2

1SOPY: RETURN TO

GWL (NLIL-2)

AND AFB, N MEX

\title{
ON THE MICHAILOV CRITERION FOR EXPONENTIAL POLYNOMIALS
}

by Allan M. Krall

\section{Prepared by}

PENNSYLVANIA STATE UNIVERSITY

University Park, Pa.

for

NATIONAL AERONAUTICS AND SPACE ADMINISTRATION - WASHINGTON, D. C. - MAY 1966 
NASA LiR-40Y

ON THE MICHAILOV CRITERION FOR EXPONENTIAL POLYNOMIALS

By Allan M. Krall

Distribution of this report is provided in the interest of information exchange. Responsibility for the contents resides in the author or organization that prepared it.

Prepared under Contract No. NGR-39-009-041 by PENNSYLVANIA STATE UNIVERSITY University Park, Pa.

for

\section{NATIONAL AERONAUTICS AND SPACE ADMINISTRATION}

For sale by the Clearinghouse for Federal Scientific and Technical Information Springfield, Virginia 22151 - Price $\$ 1.00$ 
On the Michailov Criterion for Exponential Polynomials

$$
\text { by }
$$

$$
\text { Allan M. Krall }{ }^{1}
$$

We consider an equation of the form

$$
F(z)=\sum_{i=1}^{m} \sum_{j=0}^{n} \tilde{a}_{i j} z^{j} e^{\omega_{i} z}=0
$$

where $0 \leqq \omega_{1}<\omega_{2} \cdots<w_{m}$ are real numbers and $\tilde{a}_{1 j}, i=1, \ldots m, j=1, \ldots$ n are complex numbers. We assume that there is at least one coefficient $a_{1 j}$ different from zero when $1 \geqq 1$.

In many applications it is necessary to know whether or not $F(z)$ has any zeros in the right half plane. The best known technique for answering this question is due to Pontrjagin [1] and [2]. Pontrjagin's criterion, however, is very difflcult to apply. In fact it has been found unsatisfactory except as a theoretical result (see [3, page 420]). Popov, [3, page 420]), states that to provide a more useful criterion, A. A. Sokolov [4] and N. N. Miasnikov extended the Michailov criterion to cover exponential polynomials such as $F(z)$. Unfortunately no reference is given for

$\overline{1}$

McAllister Bullding, The Pennsylvania State University, University Park, Pennsylvania. 
Miasnikov's work and Sokolov's is inaccessible in this country. Since the Michailov criterion is easily stated, easily proved and easy to apply, it seems advantageous even at the risk of duplication to present it here. Dividing by $e^{w_{m}}$ and letting $\widetilde{a}_{i j}=a_{m-i+1, j}$ we transform (1) into

$$
G(z)=\sum_{i=1}^{m} \sum_{j=0}^{n} a_{i j} z^{j} e^{-r_{i} z}=0
$$

Here $r_{i}=w_{m}-w_{m-i+1}>0$ for $i=2,3, \ldots m$ and $r_{1}=0$. The zeros of $F(z)$ and $G(z)$ are the same, and it is $G(z)$ we will consider from now on.

In general $G(z)$ will contain an infinite number of zeros which occur in chains having the following properties.

1. The imaginary parts of the zeros are $O(k)$ for $k= \pm 1, \pm 2, \ldots \pm n, \ldots$

2. The real parts of the zeros are O(Iog k), (advanced type), constant, (neutral type) or - o(log k) (retarded type).

Any combination of advanced, neutral or retarded types may occur.

The term $a_{\ln } z^{n}$ is said to be the principle term of $G(z)$. If the principle term is present, there are no zeros of advanced type. However, there still may be 
an infinite number of zeros in the right half plane because of the presence of zeros of neutral type. We will need the following hypotheses in what follows.

$$
\begin{aligned}
& \text { H 1. }\left|a_{1 n}\right|>\sum_{i=2}^{m}\left|a_{i n}\right| . \\
& H \text { 2. } a_{i n}=0,1=2,3, \ldots m .
\end{aligned}
$$

H 3. All the coefficients $a_{1 j}$ are real.

Theorem 1. Let $\mathrm{H} I$ hold. Let

$$
\begin{aligned}
& D=\left|a_{1 n}\right|-\sum_{i=2}^{m}\left|a_{i n}\right|, \\
& M=\sup _{j=0,1, \ldots n-1} \sum_{i=2}^{m}\left|a_{i j}\right| / D 。
\end{aligned}
$$

Then in the right half plane $G(z)$ has at most a finite number of zeros all of which lie inside a semicircle of radius $M+I$ centered at the origin.

Proof, Let $|z|>M+1$. Then

$\left.|G(z)| \geqq\left|a_{I n}\right||z|^{n}-\sum_{i=2}^{m}\left|a_{i n}\right||z|^{n}-\sum_{j=0}^{n-1} \sum_{i=1}^{m}\left|a_{1 j}\right|\right)|z|^{j}$ 


$$
\begin{aligned}
& \geqq D\left[|z|^{n}-M \sum_{j=0}^{n-I}|z|^{j}\right] \\
& \geqq D\left[|z|^{n}-M\left(|z|^{n}-1\right) /(|z|-1)\right] \\
& \geqq D\left[|z|^{n}\{|z|-(M+1)\}+M\right] /(|z|-1) \\
& >0 .
\end{aligned}
$$

If $G(z)$ had an infinite number of zeros in the right half plane, they would approach infinity giving a contradiction.

We write

$$
G(z)=a_{\ln } z^{n}(1+\phi(z)+\psi(z)),
$$

where

and

$$
\phi(z)=\sum_{i=2}^{m}\left(a_{i n} / a_{l n}\right) e^{-r_{i} z}
$$

$$
\Psi(z)=\sum_{i=2}^{m} \sum_{j=0}^{n-1}\left(a_{1 j} / a_{1 n}\right) z^{j-n} e^{-r_{1} z} .
$$

Note that in the right half plane including the imaginary axis $|\phi(z)|<I$ and $\Psi(z)=O(I)$ as $|z| \rightarrow \infty$ whenever $H I$ holds.

Theorem 2. (Michailov Criterion). Let $\mathrm{H} 1$ hold. As $z$ varies along the imaginary axis from - $1 \infty$ to 1 o then $G(z)$ passes through the origin each time $G(z)$ has an Imaginary zero. If $G(z)$ has no imaginary zeros, then 


$$
\begin{aligned}
N & =\quad \frac{n}{2}-\frac{1}{2 \pi} \Delta(-1 y, i y) \arg G(z)+(8) \\
& +\frac{1}{2 \pi}[\arg (1+\phi(i y)+\Psi(i y))-\arg (1+\phi(-1 y) \\
& +\Psi(-i y))]
\end{aligned}
$$

where $N$ is the number of zeros of $G(z)$ in the right half plane, $\mathrm{y}$ is any number greater than $M+1$ of theorem 1 , large enough so that $|\phi(z)+\Psi(z)|<1$ when $|z| \geq y$ and $\Delta(1 y,-1 y)$ arg $G(z)$ is the net change in arg $G(z)$ as $z$ varies from -iy to iy.

Proof. We choose a semicircular contour $C$ varying from -iy to iy along a circle centered at the origin with radius $y$ and then along the imaginary axis from iy to -iy. If $G(z)$ has no zeros on the imaginary axis then it is wel.1 known that

$$
N=\frac{1}{2 \pi} \Delta_{c} \arg G(z)-\frac{1}{2 \pi} \Delta(-i y, i y) \arg G(z)
$$

where $\Delta_{c}$ denotes the net change in arg $G(z)$ along the semicircle. We see from (7) that

$$
\begin{aligned}
\Delta_{c} \arg G(z)=n \Delta_{c} & \arg z+\Delta_{c} \arg (1+\phi(z)+\Psi(z)) . \\
\Delta_{c} \arg G(z)=n \pi & +\arg (1+\phi(1 y)+\Psi(1 y))- \\
& -\arg (1+\phi(-1 y)+\Psi(-1 y))
\end{aligned}
$$

since $I+\phi(z)+\Psi(z)$ cannot wind around the origin. Inserting (11) in (9) achieves the result. 
Corollary 1. If $H 1$ holds, and $G(z)$ has no imaginary zeros, then $\mathrm{N}=0$ if and only if

$$
\begin{aligned}
\Delta(-1 \mathrm{y}, \mathrm{iy}) \arg G(z)=n \pi & +\arg (1+\phi(1 \mathrm{y})+\Psi(1 \mathrm{y}))- \\
& -\arg (1+\phi(-1 \mathrm{y})+\Psi(-1 \mathrm{y})
\end{aligned}
$$

Corollary 2. If $\mathrm{H} I$ and $\mathrm{H} 3 \mathrm{hold}$, and $\mathrm{G}(\mathrm{z})$ has no imaginary zeros, then

$$
N=\frac{n}{2}-\frac{1}{\pi} \Delta(0,1 y) \arg G(z)+\frac{1}{\pi} \arg (I+\phi(1 y)+\psi(1 y)) .
$$

Proof. When $\mathrm{H} 3$ holds the argument for $\overline{\mathrm{y}}$ are the negative of those for $y$.

Corollary 3. If $\mathrm{H} I$ and $\mathrm{H} 3$ hold, and $\mathrm{G}(\mathrm{z})$ has no imaginary zeros, then $N=0$ if and only if

$$
\Delta(0, \text { i.y }) \arg G(z)=\frac{n \pi}{2}+\frac{1}{\pi} \arg (1+\phi(1 y)+\Psi(1 y)) .
$$

Corollary 4. If $\mathrm{H} I$ and $\mathrm{H} 2$ hold, and $\mathrm{G}(\mathrm{z})$ has no imaginary zeros, then

$$
N=\frac{n}{2}-\frac{1}{2 \pi} \Delta(-1 \infty, 1 \infty) \arg G(z)
$$

Proof. When H 2 holds, $\phi(z) \equiv 0$. Since $\Psi(z)=O(1), 1+\Psi(z) \rightarrow 1$ as $|z| \rightarrow \infty$. Thus the last two terms of (8) approach 0 .

Corollary 5. If $\mathrm{H} I$ and $\mathrm{H} 2$ hold, and $G(z)$ has no imaginary zeros, then $N=0$ if and only if 


$$
\Delta(-1 \infty, 1 \infty) \arg G(z)=n \pi
$$

Corollary 6. If $\mathrm{H}, \mathrm{H} 2$ and $\mathrm{H} 3$ hold, and $\mathrm{G}(\mathrm{z})$ has no Imaginary zeros, then

$$
N=\frac{n}{2}-\frac{1}{\pi} \Delta(0,1 \infty) \arg G(z)
$$

Corollary 7. If $\mathrm{H} 1, \mathrm{H} 2$ and $\mathrm{H} 3$ hold, and $\mathrm{G}(\mathrm{z})$ has no Imaginary zeros, then $N=0$ if and only if

$$
\Delta(0,1 \infty) \text { arg } G(z)=\frac{n \pi}{2}
$$

If these statements are compared to those of Pontrjagin, it is easy to see that they imply each other. The main advantage to the Michailov criterion is the rew moval of the perpetual oscillation present in Pontrjagin's criterion.

It is a fairly simple matter to see if arg $G(z)$ varies through the appropriate number of quadrants or not. This procedure should prove quite useful in application. 


\section{REFERENCES}

1. Richard Bellman and Kenneth I. Cooke, "Differential Difference Equations", Academic Press, New York, 1963.

2. L. S. Pontrjagin, "On the Zeros of Some Elementary Trancendental Functions", Amer. Math. Soc. Trans., Ser. 2, Vol. I, 1955, pp. 95-110.

3. E. P. Popov, "The Dynamics of Automatic Control Systems", Addison-Wesley, Reading, Mass., 1962.

4. A. A. Sokolov, "Kriteri1 ustolchivosti lineinykh sistem regulirovanila s raspredelennymi parametrami $i$ ego primenenila. (The stability criterion in Iinear regulation systems with distributed parameters and 1ts application)," Inzhenerny1 Sbornik 2,2 (1946). 